\title{
Mechanistic insights into ventricular arrhythmogenesis of hydroxychloroquine and azithromycin for the treatment of COVID-19
}

Gongxin Wang ${ }^{1}$, Chieh-Ju Lu ${ }^{1}$, Andrew Trafford ${ }^{2}$, Xiaohui Tian ${ }^{3}$, Hannali Flores ${ }^{4}$, Piotr Maj $^{5}$, Kevin Zhang ${ }^{6}$, Yanhong Niu ${ }^{7}$, Luxi Wamg ${ }^{1}$, yimei $\mathrm{Du}^{8}$, Xinying $\mathrm{Ji}^{9}$, Yanfang $\mathrm{Xu}^{10}$, Lin $\mathrm{Wu}^{11}$, Dan $\mathrm{Li}^{12}$, Herring Neil ${ }^{5}$, David Paterson ${ }^{12}$, Christopher Huang ${ }^{13}$, Henggui Zhang $^{4}$, Ming Lei ${ }^{12}$, and Guoliang $\mathrm{Hao}^{1}$

${ }^{1}$ Henan SCOPE Research Institute of Electrophysiology Co. Ltd.

${ }^{2}$ Manchester University

${ }^{3}$ Huaihe Hospital, Henan University

${ }^{4}$ The University of Manchester

${ }^{5}$ University of Oxford

${ }^{6}$ Imperial College London

${ }^{7}$ Fuwai Central China Cardiovascular Hospital

${ }^{8}$ Union Hospital

${ }^{9}$ Henan University

${ }^{10}$ Hebei Medical University

${ }^{11}$ Peking University First Hospital

${ }^{12}$ Uinversity of Oxford

${ }^{13}$ University of Cambridge

June 5, 2020

\begin{abstract}
Background and Purpose: We investigate mechanisms for potential pro-arrhythmic effects of hydroxychloroquine (HCQ) alone, or combined with azithromycin (AZM), in Covid-19 management supplementing the limited available experimental cardiac safety data. Experimental Approach: We integrated patch-clamp studies utilizing In Vitro ProArrhythmia Assay Schema IC50 paradigms, molecular modelling, cardiac multi-electrode array and voltage (RH237) mapping, ECG studies, and Ca2+ (Rhod-2 AM) mapping in isolated Langendorff-perfused guinea-pig hearts with human in-silico ion current modelling. Key Results: HCQ blocked IKr and IK1 with IC50s $(10 \pm 0.6$ and $34 \pm 5.0 \mu \mathrm{M})$ within clinical therapeutic ranges, INa and ICaL at higher IC50s, leaving Ito and IKs unaffected. AZM produced minor inhibition of INa, ICaL, IKs, and IKr,, sparing IK1 and Ito. HCQ+AZM combined inhibited IKr and IK1 with IC50s of $7.7 \pm 0.8 \mu \mathrm{M}$ and 30.4 $\pm 3.0 \mu \mathrm{M}$, sparing INa, ICaL and Ito. Molecular modelling confirmed potential HCQ binding to hERG. HCQ slowed heart rate and ventricular conduction. It prolonged PR, QRS and QT intervals, and caused prolonged, more heterogeneous, action potential durations and intracellular Ca2+ transients. These effects were accentuated with combined HCQ+AZM treatment, which then elicited electrical alternans, re-entrant circuits and wave break. Modelling studies attributed these to integrated HCQ and AZM actions reducing IKr and IK1, thence altering cell $\mathrm{Ca} 2+$ homeostasis. Conclusion and implications: Combined HCQ+AZM treatment exerts pro-arrhythmic ventricular events by synergetically inhibiting IKr, IKs with resulting effects on cellular Ca2+ signalling, and action potential propagation and duration. These findings provide an electrophysiological basis for recent FDA cardiac safety guidelines cautioning against combining HCQ/AZM when treating Covid-19.
\end{abstract}


Mechanistic insights into ventricular arrhythmogenesis of hydroxychloroquine and azithromycin for the treatment of COVID-19

Authors:

Gongxin Wang ${ }^{1}$, Chieh-Ju Lu${ }^{1}$, Andrew W. Trafford ${ }^{2}$, Xiaohui Tian ${ }^{3}$, Hannali M Flores ${ }^{4}$, Piotr Maj ${ }^{5}$, Kevin Zhang ${ }^{6}$, Yanhong $\mathrm{Niu}^{7}$, Luxi Wang ${ }^{1}$, Yimei $\mathrm{Du}^{8}$ Xinying $\mathrm{Ji}^{3}$, Yanfang $\mathrm{Xu}^{9}$, Lin $\mathrm{Wu}^{10}$, $\mathrm{Dan} \mathrm{Li}^{11}$, Neil Herring ${ }^{11}$, David Paterson ${ }^{11}$, Christopher L.-H. Huang ${ }^{12,14}$, Henggui Zhang ${ }^{4,12,14,}$, Ming Lei ${ }^{5}$, Guoliang $\mathrm{HaO}^{1,11}$

Affiliations:

${ }^{1}$ Henan SCOPE Research Institute of Electrophysiology Co. Ltd., China

${ }^{2}$ Unit of Cardiac Physiology, Institute of Cardiovascular Sciences, Manchester Academic Health Sciences Centre, The University of Manchester, UK.

${ }^{3}$ Department of Pharmacy, Huaihe Hospital and College of Medicine, Henan University, China.

${ }^{4}$ Biological Physics Group, Department of Physics and Astronomy, The University of Manchester, UK

${ }^{5}$ Department of Pharmacology, University of Oxford, Oxford, UK

${ }^{6}$ School of Medicine, Imperial College of London, UK

${ }^{7}$ Fuwai Central China Cardiovascular Hospital, Zhengzhou, China

${ }^{8}$ Department of Cardiology, Union Hospital, Tongji Medical College, Huazhong University of Science and Technology, Wuhan, China

${ }^{9}$ Department of Pharmacology, Hebei Medical University, China

${ }^{10}$ Department of Cardiology, Peking University First Hospital, Beijing, China

${ }^{11}$ Department of Physiology, Anatomy and Genetics, University of Oxford, Oxford, UK

${ }^{12}$ Physiological Laboratory and Department of Biochemistry, University of Cambridge, UK

${ }^{13}$ Peng Cheng Laboratory, Shenzhen, China

${ }^{14}$ Key Laboratory of Medical Electrophysiology of the Ministry of Education and Institute of Cardiovascular Research, Southwest Medical University, Luzhou, China

Short title:

Pro-arrhythmic actions of hydroxychloroquine and azithromycin

Correspondence:

Dr. Gaoliang Hao, Henan SCOPE Research Institute of Electrophysiology Co. Ltd., Kaifeng

475000, China and Department of Physiology, Anatomy and Genetics, University of Oxford, Tel: 008613552324220, Fax: 008613552324220, E-mail: guoliang.hao@epscopelab.com

Or Prof. Ming Lei, Department of Pharmacology, University of Oxford, Mansfield Road, Oxford OX1 3QT, United Kingdom. Tel: 00441865271850; Fax: 00441865271850 E-mail: ming.lei@pharm.ox.ac.uk

CLHH, HZ, ML and GLH are joint senior authors

Length of Main text: 3198

Abstract

Background and Purpose: 
We investigate mechanisms for potential pro-arrhythmic effects of hydroxychloroquine (HCQ) alone, or combined with azithromycin (AZM), in Covid-19 management supplementing the limited available experimental cardiac safety data.

Experimental Approach:

We integrated patch-clamp studies utilizing In Vitro ProArrhythmia Assay (CiPA) Schema $\mathrm{IC}_{50}$ paradigms, molecular modelling, cardiac multi-electrode array and voltage (RH237) mapping, ECG studies, and $\mathrm{Ca}^{2+}$ (Rhod-2 AM) mapping in isolated Langendorff-perfused guinea-pig hearts with human in-silico ion current modelling.

Key Results:

HCQ blocked $\mathrm{I}_{\mathrm{Kr}}$ and $\mathrm{I}_{\mathrm{K} 1}$ with $\mathrm{IC}_{50} \mathrm{~s}(10 \pm 0.6$ and $34 \pm 5.0 \mu \mathrm{M})$ within clinical therapeutic ranges, $\mathrm{I}_{\mathrm{Na}}$ and $\mathrm{I}_{\mathrm{CaL}}$ at higher $\mathrm{IC}_{50} \mathrm{~s}$, leaving $\mathrm{I}_{\mathrm{to}}$ and $\mathrm{I}_{\mathrm{Ks}}$ unaffected. AZM produced minor inhibition of $\mathrm{I}_{\mathrm{Na}}, \mathrm{I}_{\mathrm{CaL}}, \mathrm{I}_{\mathrm{Ks}}$,

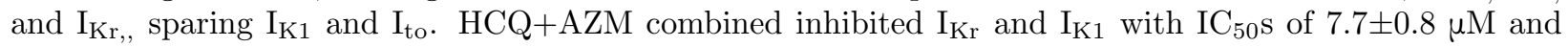
$30.4 \pm 3.0 \mu \mathrm{M}$, sparing $\mathrm{I}_{\mathrm{Na}}, \mathrm{I}_{\mathrm{CaL}}$ and $\mathrm{I}_{\mathrm{to}}$. Molecular modelling confirmed potential HCQ binding to hERG. HCQ slowed heart rate and ventricular conduction. It prolonged PR, QRS and QT intervals, and caused prolonged, more heterogeneous, action potential durations and intracellular $\mathrm{Ca}^{2+}$ transients. These effects were accentuated with combined HCQ+AZM treatment, which then elicited electrical alternans, re-entrant circuits and wavebreak. Modelling studies attributed these to integrated HCQ and AZM actions reducing $\mathrm{I}_{\mathrm{Kr}}$ and $\mathrm{I}_{\mathrm{K} 1}$, thence altering cell $\mathrm{Ca}^{2+}$ homeostasis.

Conclusion and implications:

Combined HCQ+AZM treatment exerts pro-arrhythmic ventricular events by synergetically inhibiting $\mathrm{I}_{\mathrm{Kr}}$, $\mathrm{I}_{\mathrm{Ks}}$ with resulting effects on cellular $\mathrm{Ca}^{2+}$ signalling, and action potential propagation and duration. These findings provide an electrophysiological basis for recent FDA cardiac safety guidelines cautioning against combining HCQ/AZM when treating Covid-19.

Key words: Cardiac safety; Ion channels, Hydroxychloroquine; Azithromycin, CiPA, COVID-19

\section{Introduction}

The severe acute respiratory( syndrome coronavirus 2 (SARS-CoV-2) pandemic has now infected $>6.34$ million individuals causing 380810 deaths as of 3-June-2020 (www.ecdc.eurpoe.eu). Although there are no approved drugs to prevent or treat SARS-CoV-2 infection, recent use of the anti-malarial 4-aminoquinolines chloroquine (CQ) or hydroxychloroquine (HCQ) alone, or combined with the antibiotic azithromycin (AZM) in Covid-19 therapy has attracted global attention and extensive early clinical use and trials(NIH, 2020; Pharmafield, 2020). An initial nonrandomized open label clinical trial of 20 cases, had associated HCQ treatment with viral load reduction or disappearance in COVID-19 patients, effects reinforced by AZM(Gautret et al., 2020). This subsequently prompted the US Food and Drugs Administration (FDA) authorization of emergency use of CQ or HCQ, alone or in combination with AZM in Covid-19 therapy(Hinton, 2020; Lenzer, 2020). Currently, large number of trials of these combinations, with or without the addition of further drugs, are registered worldwide (NIH, 2020; Pharmafield, 2020).

However, several recent studies have flagged cardiac safety concerns for the CQ/HCQ and AZM regime in Covid-19 therapy. Particular concern has arisen regarding potential synergistic effects of HCQ and AZM on QT duration and cardiovascular mortality (Borba et al., 2020; Chorin et al., 2020; Lane et al., 2020; Magagnoli et al., 2020; Mercuro et al., 2020). Higher mortalities and incidences of prolonged corrected QT (QTc) intervals were reported in patients at a Brazilian hospital receiving the high dose $(12 \mathrm{~g}$ total dose over 10 days, $\sim 1.2 \mathrm{~g}$ /day, giving plasma levels of $\sim 10 \mu \mathrm{M}$ ) than the lower dose of CQ, typified by its use in other conditions such as rheumatoid arthritis and systemic lupus erythematosus (SLE). This phase $2 \mathrm{~b}$ randomized Covid-19 trial was terminated after enrolment of only 81 out of the target of 440 patients owing to occurrences of adverse events (Borba et al., 2020). A retrospective data analysis from United States Veterans Health Administration medical centers suggested increased overall mortality even in patients treated with HCQ alone 
(Magagnoli et al., 2020). The largest available analysis from an international consortium on HCQ safety covering over 950,000 patients from six countries declared HCQ safe for short-term use at doses currently used for conditions such as rheumatoid arthritis. However, it urged caution in its use when combined with AZM in view of potential risks of cardiovascular mortality related to QTc prolongation (Lane et al., 2020). Two recent reports of 90 and 201 patients hospitalized with COVID-19 treated with HCQ, with or without AZM, associated these drugs with QTc prolongation, especially when used in combination (Mercuro et al., 2020; Saleh et al.). Although these studies are small, only one case of torsades de pointes was documented and the therapy was seldom discontinued. They suggested that more QT interval monitoring studies should be undertaken before final recommendations can be made(Saleh et al.). Based on these findings, the FDA issued a cautions warning against use of HCQ or CQ for COVID-19 outside of the hospital setting or a clinical trial, due to risks of heart rhythm problems(Hinton, 2020). This does not affect FDA-approved uses in malaria, systemic lupus erythematosus (SLE), and rheumatoid arthritis where they have been used many years. Both HCQ and AZM have been in clinical use alone or in combination in other disease conditions for many years with cardiac safety then not considered a major concern.

Currently, there is very limited data evaluating cardiac safety particularly concerning use of HCQ in combination with AZM, although multiple randomized trials are currently in progress evaluating the efficacy of this combination for Covid-19. We therefore explored the cardiac safety of HCQ alone or in combination with AZM using the FDA recommended Comprehensive in Vitro Proarrhythmia Assay (CiPA) protocols to understand arrhythmic mechanisms (Strauss et al., 2018). Furthermore, we expanded the CiPA protocol by introducing assessments of effects on membrane and $\mathrm{Ca}^{2+}$ clocks, their interaction and cardiac conduction recently suggested as crucial indicators for ventricular arrhythmogenesis (Lei \& Huang, 2019; Lei, Wu, Terrar \& Huang, 2018).

\section{Methods and Materials}

\subsection{Study Approval}

All procedures were approved by The Institutional Animals Ethics Committees at Henan University, Kaifeng, China under and the national guidelines under which the institution operates, and also conform with the NIH Guide for the Care and Use of Laboratory Animals. Guinea pigs (male; 300-350 g) were provided by The Experimental Animal Center of the Medical College of Xi'an Jiaotong University and maintained in a pathogen-free facility at Henan University with ad libitum access to food and water. Animals were humanely sacrificed by intraperitoneal injection with $3 \%$ pentobarbital sodium $(50 \mathrm{mg} / \mathrm{kg})$.

\subsection{Single-cell electrophysiological recordings}

Core human cardiac channels were heterologously expressed in recombinant HEK293 or CHO cell lines and studied using conventional patch clamp methods. Stable cell lines expressing Nav1.5 and Cav1.2/ $/ 22 / \alpha 2 / \delta 1$, hERG, Kir2.1, Kv4.3, KCNQ1/E1 were used. All currents were recorded using a MultiClamp 700A amplifier, 1550A digitizer and pCLAMP 10.6 software (Molecular devices, USA). Patch electrodes with resistances of 2-5 M were pulled with a P-97 micropipette puller (Sutter Instruments, USA). The conventional whole-cell recordings were obtained by manual suction for hERG, Kir2.1, Kv4.3 and Nav1.5 studies. Perforated wholecell recordings of KCNQ1/E1 and Cav1.2 were obtained following perforation with $200 \mu \mathrm{g} / \mathrm{ml}$ Amphotericin included in the pipette solution. After whole-cell mode were obtained, cells were used in voltage clamp mode for the current recordings. The external solution contained $(\mathrm{mM}): \mathrm{NaCl} 137 ; \mathrm{MgCl}_{2} \quad 1 ; \mathrm{KCl} 4 ;$; glucose 10; HEPES $10 ; \mathrm{CaCl}_{2} 1.8, \mathrm{pH}$ adjusted to 7.4 with $\mathrm{NaOH}$. The $\mathrm{K}^{+}$-containing pipette solution contained $(\mathrm{mM}): \mathrm{KCl} 40 ; \mathrm{KF} 100 ; \mathrm{MgCl}_{2}$ 2; EGTA 5;HEPES 10,pH adjusted to 7.2 with $\mathrm{KOH}$. The sodium and calcium current pipette solution contained (mM): $\mathrm{CsCl} 65 ; \mathrm{CsF} 75 ; \mathrm{MgCl}_{2} 2.5$;EGTA 5;HEPES 10, pH adjusted to 7.3 with $\mathrm{CsOH}$.

\subsection{Molecular modelling and docking studies}

The experimental structure of the open hERG channel (PDB ID: 5va2) was selected as a receptor for molecular docking studies. The structure was prepared for docking with Protein Preparation Wizard on 
default settings including modelling of missing loops using Prime. The centroid of residues Y652 from every subunit was defined as the centre of the docking binding site. HCQ and AZM structures were obtained from PubChem and prepared for docking using LigPrep. The recently proposed Induced Fit Docking (IFD) protocol was used (Negami, Araki, Okuno \& Terada, 2019). Protein Preparation Wizard, LigPrep, Prime, and IFD were executed as parts of the Maestro software suite 2018-4 (Schrödinger, LLC, NY).

\subsection{Langendorff- perfused isolated hearts}

Guinea pigs (male; 300-350 g) were humanely killed by intraperitoneal injection with pentobarbital sodium $(50 \mathrm{mg} / \mathrm{kg})$. Hearts rapidly excised after thoracotomy were mounted onto a Langendorff perfusion system and perfused with a modified Tyrode's solution $\left(119 \mathrm{NaCl}, 25 \mathrm{NaHCO}_{3}, 4 \mathrm{KCl}, 1.2 \mathrm{KH}_{2} \mathrm{PO}_{4}, 1 \mathrm{MgCl}_{2}\right.$, $1.8 \mathrm{CaCl}_{2} \cdot 2 \mathrm{H}_{2} \mathrm{O}$, and $10 \mathrm{D}$-glucose $(\mathrm{mM})$ equilibrated with $5 \% \mathrm{CO}_{2}$ and $\left.95 \% \mathrm{O}_{2}\right)$ with the flow rate of 8 $\mathrm{ml} / \mathrm{min}$ at $37^{\circ} \mathrm{C}$. Hearts were perfused and monitored for stability for 20 mins before experimental procedures commenced.

\subsection{Electrical mapping of ex vivo heart preparations}

A multi-electrical array (MEA) mapping system (EMS64-USB-1003, MappingLab Ltd., UK) was employed to record extracellular potentials (ECP) from the epicardium. Two 64 channel MEAs (MappingLab Ltd., UK) were employed for left atrial and left ventricular simultaneous recordings. ECP signals were recorded at a $10 \mathrm{kHz}$ sampling using synchronized 64 channel EMS64-USB-1003 recording systems and recorded using EMapRecord 5.0 software (MapingLab Ltd., UK) and stored in a computer for subsequent off-line analysis. Electrocardiograms (ECG) were continuously recorded by two ECG electrodes (MappingLab Ltd., UK) positioned on the right atrium and left ventricle respectively.

The epicardial isochronal activation map during sinus rhythm or imposed pacing was plotted as time differences between the earliest activation site and the individual activation site on each channel. Activation times were calculated as the point of maximal negative slope of activation waveforms. The conduction velocity $(\mathrm{CV})$ was calculated from the difference in timing and the known distance between the recording points using EMapScope 5.0 software (MappingLab Ltd.).

\subsection{Optical mapping of ex vivo heart preparations}

After the Langendorff-perfused hearts reached steady state, contraction artefacts were mimimised using blebbistatin $(10 \mu \mathrm{M})$. Dye loading was aided by pre-perfusion with pluronic F127 (20\% w/v in DMSO). $\operatorname{Rh} 237(1 \mu \mathrm{g} / \mathrm{ml})$ and Rhod2-AM $(1 \mu \mathrm{g} / \mathrm{ml})$ were perfused to enable simultaneous membrane potential and $\mathrm{Ca}^{2+}$ measurements at 37 .

Two $530 \mathrm{~nm}$ LEDs (LEDC-2001, MappingLab Ltd) were used to illuminate the heart after their emissions were bandpass filtered (wavelengths $530 \pm 20 \mathrm{~nm}$ ) to minimize stray excitation light reaching the dyes. The fluorescence light was passed through a $550 \mathrm{~nm}$ long-pass filter and then a dichroic mirror with a cut off of $638 \mathrm{~nm}$. Fluorescence light with wavelengths above $638 \mathrm{~nm}$ was passed through a $700 \mathrm{~nm}$ long-pass filter and then imaged by the camera for recording voltage signals. Fluorescence light below $638 \mathrm{~nm}$ was passed through a bandpass filter $(585+-40 \mathrm{~nm})$ then imaged by the camera for recording calcium signals (OMSPCIE-2002, MappingLab Ltd). The raw spatial resolution was 128-by-128 pixels, the total mapping area was $16 \times 16 \mathrm{~mm}$ and the temporal resolution was 900 frames/second.

The cameras of the optical mapping system, LED lights and stimulator and ECG recording were simultaneously driven by an 8 channel TTL analog-digital converter and OMapRecord 4.0 software (MappingLab Ltd.). For the analysis of optical mapping signals and generation of isochronal maps, data were semi-automatically processed using OMapScope 5.0 software (MappingLab Ltd). In this experimental configuration, stable recordings for voltage $\left(V_{\mathrm{m}}\right)$ and intracellular $\mathrm{Ca}^{2+}(\mathrm{CaT})$ signals could be obtained for $>2$ hours in preliminary experiments assessing system stability.

\subsection{Arrhythmia induction protocol}


Stimuli were generated by an isolated constant voltage/current stimulator (VCS3001, MappingLab Ltd., UK). They were delivered with a platinum electrode onto the epicardial apex at an amplitude $1.5 \mathrm{x}$ the diastolic voltage threshold and a $2 \mathrm{~ms}$ pulse width. The optical mapping was performed in conjunction with an "alternans mapping" protocol. The heart was consecutively paced at basic cycle lengths (BCL) from 210 with $10 \mathrm{~ms}$ decrement until $170 \mathrm{~ms}$. The heart was paced 50 times with $2 \mathrm{~ms}$ duration pulses and the optical mapping recording was performed at the last 2 second in each episode. This protocol was performed both before and after drugs were delivered.

\subsection{In silico model reconstruction}

The O'Hara-Rudy (ORd) human ventricular model(O'Hara, Virag, Varro \& Rudy, 2011) was modified to incorporate the actions on multi-channel block of HCQ, including the dose-dependent block of $\mathrm{I}_{\mathrm{Na}}, \mathrm{I}_{\mathrm{CaL}}$, , $\mathrm{I}_{\mathrm{to}}, \mathrm{I}_{\mathrm{Kr}}, \mathrm{I}_{\mathrm{Ks}} \mathrm{I}_{\mathrm{K} 1}$ and the $\mathrm{Na}^{+}{ }_{-} \mathrm{Ca}^{2+}$ exchange current, $\mathrm{I}_{\mathrm{NCX}}$. This model was selected as it was developed from and validated against experimental data from human ventricular cells and found suitable for studying actions of drugs. The simulations evoked action potentials by a supra-threshold stimulus in control runs and following application of HCQ, and combined application of HCQ and AZM. Action potential time courses characterized by $\mathrm{AP}$ durations at $90 \%$ repolarisation $\left(\mathrm{APD}_{90}\right)$ and maximal upstroke velocity (MUP) and the underlying ion channel currents targeted by the drugs were recorded for analysis.

\subsection{Statistical analysis}

All patch clamp recorded data were analyzed using Clampfit 10.6 (Molecular Devices, USA), OriginPro 8.0 (Origin Lab, USA) and Adobe Illustrator 10 (Adobe, USA). The concentration-response curve was fitted by the logistic equation $y=A_{2}+\left(A_{1}-A_{2}\right) /\left[1+\left(x / x_{0}\right)^{p}\right]$, where $x$ is the drug concentration, and $p$ is the Hill coefficient. All data are expressed as the means +- SEM. One-way ANOVA followed by multiple-comparison test was used to evaluate multiple test treatments. A value of $\mathrm{p}<0.05$ was considered to be statistically significant. $\mathrm{IC}_{50}$ denotes the concentration determined for half-maximal inhibitory effects. In the figures, the designations for $\mathrm{p}$ values are: ${ }^{*} \mathrm{p}<0.05,{ }^{* *} \mathrm{P}<0.01$ and ${ }^{* * *} \mathrm{P}<0.001$ respectively.

\section{Results}

The four groups of experiments conducted assessed the mechanistic basis for the clinical reports bearing upon cardiac safety of HCQ, whether applied alone or in combination with AZM.

\subsection{Patch-clamp studies of single channel characteristics}

The first series of experiments assessed the effects of HCQ (Fig. 1) and AZM alone (Fig. 2) and in combination (Fig. 3) on a range of human cardiac ion channels. The experiments on HCQ and AZM themselves adopted concentrations fully encompassing plasma levels associated with their clinical use. The same HCQ concentration range was then combined with $10 \mu \mathrm{M}$ AZM. These examined inward, cardiac channel currents from hNav1.5 $\left(\mathrm{I}_{\mathrm{Na}}, A\right)$, Cav1.2 $\left(\mathrm{I}_{\mathrm{Ca}, \mathrm{L}}, B\right)$, mediating or maintaining membrane action potential depolarization. They also studied outward Kv4.3 ( $\left.\mathrm{I}_{\mathrm{to}}, C\right)$, hERG $\left(\mathrm{I}_{\mathrm{Kr}}, C\right)$, KCNQ1/E1 ( $\mathrm{I}_{\mathrm{Ks}}, E$ ) and Kir2.1 ( $\left.\mathrm{I}_{\mathrm{K} 1}, F\right)$ normally mediating action potential recovery. This encompassed the range of ion channels recommended by the CiPA protocol.

Figure1 (left panels, i) exemplifies observed ionic currents in the presence of $0,1,10$ and $100 \mu \mathrm{M} H \mathrm{HCQ}$. The insets summarize the related voltage clamping protocols, fully described in extended Methods in Supplementary data. Five concentrations ranging from $10^{-8}$ to $10^{-4} \mathrm{M}$ were tested for each current examined in 3 to 6 cells at each concentration. Mean concentration-response plots (right panels, ii) used the maximum magnitudes of each current. Where applicable, the consequent alterations in such current magnitudes following pharmacological challenge were used to derive $\mathrm{IC}_{50}$ values.

Figure1 (right panels, ii) plots mean $( \pm \mathrm{SEM})$ fractional reductions in current magnitude against HCQ concentration for $\mathrm{I}_{\mathrm{Na}}(\mathrm{A}), \mathrm{I}_{\mathrm{CaL}}(\mathrm{B}), \mathrm{I}_{\mathrm{to}}(\mathrm{C}), \mathrm{I}_{\mathrm{Ks}}(\mathrm{D}), \mathrm{I}_{\mathrm{Kr}}(\mathrm{E})$ and $\mathrm{I}_{\mathrm{K} 1}(\mathrm{~F}) \cdot \mu \mathrm{M}-\mathrm{HCQ}$ inhibited both $\mathrm{I}_{\mathrm{Kr}}$ tail 
and $\mathrm{I}_{\mathrm{K} 1}$ pulse currents with $\mathrm{IC}_{50} \mathrm{~s}$ of $10 \pm 0.6 \mu \mathrm{M}(\mathrm{D})$ and $34 \pm 5 \mu \mathrm{M}(\mathrm{F})$, respectively, with more marked effects on $\mathrm{I}_{\mathrm{Kr}}$, where the $\mathrm{IC}_{50}$ fell close to expected therapeutic levels in current Covid-19 treatment regimes (Borba et al., 2020), than $\mathrm{I}_{\mathrm{K} 1}$. In contrast, $\mu \mathrm{M}-\mathrm{HCQ}$ has less effect on $\mathrm{I}_{\mathrm{Na}}$ and $\mathrm{I}_{\mathrm{CaL}}$ with $\mathrm{IC}_{50} \mathrm{~s}$ of $113 \pm$ $78 \mu \mathrm{M}$ and $209 \pm 94 \mu \mathrm{M}$ respectively, Nevertheless, HCQ caused graded $\mathrm{I}_{\mathrm{Na}}$ reductions through the entire HCQ concentration range typified in a $22 \pm 1.8 \%$ reduction at $1 \mu \mathrm{M}$. No significant inhibition of $\mathrm{I}_{\mathrm{to}}$ and $\mathrm{I}_{\mathrm{Ks}}$ by $\mu \mathrm{M}-\mathrm{HCQ}$ was observed.

Figure 2 presents corresponding results obtained with AZM applied by itself similarly encompassing clinical therapeutic levels. A previously reported dose regime administering 500, 250 and $250 \mathrm{mg} /$ day on each of three successive days gave a day 2 serum concentration of $0.22 \mu \mathrm{M}(\mathrm{Lai}$, Ho, Jain \& Walters, 2011)). However, the entire, 0-100 $\mu \mathrm{M}$ explored AZM concentration range yielded only minimal inhibitory effects. Even 10 $\mu \mathrm{M}$ and $100 \mu \mathrm{M}$ AZM reduced $\mathrm{I}_{\mathrm{Na}}(\mathrm{A}), \mathrm{I}_{\mathrm{CaL}},(\mathrm{B}) \mathrm{I}_{\mathrm{Ks}}(\mathrm{E})$ and $\mathrm{I}_{\mathrm{Kr}}(\mathrm{D})$ by $<17 \%$ and $<35 \%$ respectively and produced negligible effects on $\mathrm{I}_{\mathrm{K} 1}(\mathrm{~F})$ and no effect on $\mathrm{I}_{\mathrm{to}}(\mathrm{C})$.

Finally, Figure 3 summarizes results of applying the same range of five HCQ concentrations displayed in Fig. 1 now combined with $10 \mu \mathrm{M}$ AZM. Adding AZM did not significantly alter the effects of HCQ on $\mathrm{I}_{\mathrm{Na}}(\mathrm{A})$, $\mathrm{I}_{\mathrm{CaL}}(\mathrm{B}), \mathrm{I}_{\mathrm{to}}(\mathrm{C})$, and $\mathrm{I}_{\mathrm{Ks}}(\mathrm{E})$, but reduced the $\mathrm{IC}_{50}$ for $\mathrm{I}_{\mathrm{Kr}}\left(\mathrm{IC}_{50}=7.7 \pm 0.8 \mu \mathrm{M}\right)$ and $\mathrm{I}_{\mathrm{K} 1}(30.4 \pm 3.0 \mu \mathrm{M})$.

\subsection{Computational molecular docking determining the interaction between HCQ, AZM and hERG}

The above findings, particularly those concerning pharmacological effects on hERG, prompted computational molecular docking explorations for possible interactions between HCQ and AZM and a recent hERG homotetramer structure (PDB ID: 5va2). Fig.4 illustrates HCQ-hERG and AZM-hERG interactions derived from molecular docking. HCQ interacts predominantly with Y652 and F656 from one or more subunits (Fig.4B-D). The Induced Fit Docking (IFD) protocol enabled HCQ to enter the space between the S5 and S6 helices. Other hERG residues involved in interactions with HCQ included F557, L622, T623, S624, and S660. Docking of AZM (Figure 4E-F) indicated similar, albeit less frequent interactions. This was reflected in an approximately two-fold weaker IFD score for AZM than for HCQ (-922 and -18340 for top-scoring positions, respectively). Variability in observed docking positions of ligands could be attributed to the highly symmetric structure of the hERG channel pore enabling many equivalent positions.

\subsection{Electrical mapping and electrocardiographic studies inex vivo heart preparations}

We then explored the effects of HCQ alone and combined with AZM in the whole heart on electrophysiological activation and recovery, their electrocardiographic correlates and the consequent $\mathrm{Ca}^{2+}$ signalling processes reflecting excitation-contraction coupling.

Figure 5A summarises sites of apical stimulation, and multi-electrode array (MEA) and ECG recording in the electrical mapping experiments. Representative left atrial (LA) and left ventricular (LV) isochronal conduction maps (B) obtained in the presence of 0 - $10 \mu \mathrm{M}$ HCQ revealed a clear reduction of conduction velocity and increased conduction heterogeneities at $10 \mu \mathrm{M}$ HCQ. Synergistic inhibitory effects on conduction velocity were observed when hearts were treated with the $10 \mu \mathrm{M}$ HCQ in combination with $10 \mu \mathrm{M}$ AZM. All these effects were reversed on drug washout (C(i)). $10 \mu \mathrm{M}$ HCQ significantly decreased conduction velocities of excitation with further decreases in velocity with the $10 \mu \mathrm{M}$ HCQ and $10 \mu \mathrm{M}$ AZM combination (C(ii)).

Quantification of the corresponding electrocardiogram (ECG) traces (D(i)) demonstrated that both $10 \mu \mathrm{M}$ HCQ, and $10 \mu \mathrm{M}$ HCQ in combination with $10 \mu \mathrm{M}$ AZM, decreased heart rate $(\mathrm{HR})(\mathrm{D}(\mathrm{i}))$, and increased QT interval (D(iii)), PR interval (D(iv)) and QRS duration (D(v)). In particular, AZM when combined with HCQ augmented its effects on HR, and QT and PR interval. Together these results suggested that $10 \mu \mathrm{M}$ HCQ, and $10 \mu \mathrm{M}$ HCQ and $10 \mu \mathrm{M}$ AZM combined, exerted synergistic inhibitory effects on heart rate and conduction, and prolonged cardiac repolarisation.

\subsection{Optical mapping studies in ex vivo heart preparations}

We next investigated the effects of HCQ alone and in combination with AZM using optical fluorescence 
mapping in sinus-paced Langendorff-perfused hearts using the recording and ECG monitoring configurations illustrated in Figure 6A(i). Voltage mapping measurements of action potential initiation and conduction (Fig. $6 \mathrm{~A}(\mathrm{ii}))$ demonstrated bradycardic effects (A(iii)), visible conduction heterogeneities and slowed conduction (A(ii)) at $10 \mu \mathrm{M} \mathrm{HCQ}$, effects were augmented by combination with $10 \mu \mathrm{M}$ AZM (Fig. 6A (iv)). Similarly, optical potentiometric mapping revealed that $10 \mu \mathrm{M}$ HCQ prolonged action potential duration at $90 \%$ repolarisation $\left(\mathrm{APD}_{90}\right)(\mathrm{B}(\mathrm{i}$ and $\mathrm{ii})$ ) with $10 \mu \mathrm{M} \mathrm{HCQ}$, effects further prolonged by its combination with AZM (Fig.5B(iii)).

$\mathrm{Ca}^{2+}$ dye, Rhod-2 AM (CaD), mapping of ventricular calcium transient (CaT) characteristics demonstrated increased magnitudes of and dispersions in $\mathrm{CaD}$ durations at $90 \%$ recovery $\left(\mathrm{CaD}_{90}\right)$ (Figure $6 \mathrm{C}(\mathrm{i})$ ). The latter were attributed to changes late in the $\mathrm{CaT}$ records $\left(\mathrm{C}(\mathrm{ii})\right.$ ). The quantified $\mathrm{CaD}_{90}$ magnitudes (B(iii)) and dispersions (B(iv)) both increased with 10 but not $1.0 \mu \mathrm{M}$ HCQ. The combination of $10 \mu \mathrm{M}$ HCQ with either 1 or $10 \mu \mathrm{M}$ AZM exerted significantly greater effects than $10 \mu \mathrm{M}$ HCQ alone.

\subsection{Optical mapping assessments of arrhythmic tendency inex vivo heart preparations}

Finally, voltage dye optical mapping studies during ventricular pacing were used to assess susceptibility to arrhythmia induction before and after drug challenge in isolated perfused hearts (Fig.7A.B). Whilst $10 \mu \mathrm{M}$ HCQ did not exert detectable arrhythmic effects at the cycle lengths (CL) used, a combination of HCQ and AZM produced AP alternans at $180 \mathrm{~ms}$ CL and episodes of non-sustained torsadogenic-like VT at $170 \mathrm{~ms}$ CL in 5 out of 5 hearts. The corresponding potentiometric maps obtained during the colour coded timepoints 1-6 are represented in C. At a CL of $170 \mathrm{~ms}$, the conduction maps with HCQ+AZM demonstrated significant disruptions of the normally coherent propagation of excitation waves with evidence of excitation re-entry and wave break. Thus, the electrophysiological changes outlined above associated particularly with HCQ and AZM in combination were associated with a ventricular arrhythmic tendency.

\subsection{Computational simulations of HCQ and AZM on human ventricular action potentials}

To translate the experimental data describing the multi-ion channel blocking effects of HCQ and/or AZM to human ventricular excitation, the ORd model was implemented to reconstruct the impacts of HCQ alone and combined with AZM, on human ventricular action potentials including $\mathrm{APD}_{90}$ and maximal upstroke velocities.

Figure 8A displays predicted action potentials under control conditions and in the presence of HCQ, and HCQ and AZM combined. It was shown that with the $10 \mu \mathrm{M}$ HCQ alone, the APD was prolonged by 100 ms as compared to the control condition. With the combined action of HCQ and AZM, a synergistic action of the two drugs on APD prolongation was seen, which was manifested in a greater increase of APD90 of $\sim 300 \mathrm{~ms}$ (Figure 8B). Such an APD prolongation can be attributable to the integrated action of reduced repolarisation potassium channel currents of $\mathrm{I}_{\mathrm{Kr}}$ and $\mathrm{I}_{\mathrm{K} 1}$, and reduced depolarising currents of $\mathrm{I}_{\mathrm{Na}}, \mathrm{I}_{\mathrm{CaL}}$ and $\mathrm{I}_{\mathrm{NaCa}}$. A small reduction in action potential maximum upstroke velocities was also observed by both $\mathrm{HCQ}$ and $\mathrm{HCQ}+\mathrm{AZM}$ due to the effects on $\mathrm{I}_{\mathrm{Na}}$.

\section{DISCUSSION}

The main findings of this study are: (1) Reported therapeutic levels of HCQ blocked multiple human cardiac ion channels, particular hERG (mediating $\left.\mathrm{I}_{\mathrm{Kr}}\right)$ and Kir2.1 $\left(\mathrm{I}_{\mathrm{K} 1},\right)$ and, to a lesser degree, hNav1.5 $\left(\mathrm{I}_{\mathrm{Na}}\right)$. AZM potentiated the effects of HCQ on hERG and Kir2.1. (2) Computational molecular docking studies correspondingly predicted HCQ binding to hERG. Accordingly, (3) at the level of intact hearts, HCQ increased QT intervals as well as ventricular AP and $\mathrm{Ca}^{2+}$ transient durations and their heterogeneities. However, (4) HCQ further reduced heart rates, A-V and ventricular conduction as well as increasing conduction heterogeneities. All these effects were accentuated by combining HCQ challenge with AZM. Furthermore, (5) The HCQ+AZM combination, but not HCQ alone, then went on to increase susceptibilities to ventricular arrhythmic events consistent with significant clinical cardiac safety risks. (6) Humanin-silico modelling of ion channel effects of HCQ and AZM recapitulated the altered APDs but not the reduced conduction. This mechanistic electrophysiological data complements ongoing multiple randomized trials evaluating clinical 
efficacy and cardiac safety profiles of these drugs as a treatment for Covid-19. The findings directly align with recent FDA guidelines concerning their use.

These successive lines of investigation were combined to reach an integrated view of HCQ and AZM action. This began with the approach recommended by the CiPA guidelines (Strauss et al., 2018) in which we examined effects of HCQ and AZM on human cardiac ion channels responsible for the main inward (hNav1.5 $\left.\left(\mathrm{I}_{\mathrm{Na}}\right) \operatorname{Cav1.2}\left(\mathrm{I}_{\mathrm{Ca}, \mathrm{L}}\right)\right)$ and outward $\left(\mathrm{Kv} 4.3\left(\mathrm{I}_{\mathrm{to}}\right)\right.$, hERG $\left(\mathrm{I}_{\mathrm{Kr}}\right)$, KCNQ1/E1 $\left(\mathrm{I}_{\mathrm{Ks}}\right)$ and Kir2.1 $\left.\left(\mathrm{I}_{\mathrm{K} 1}\right)\right)$ currents in the human ventricular myocardium. HCQ and AZM were studied both alone and in combination through a wide concentration range encompassing and extending beyond reported therapeutic levels. The latter typically fell around 1.15-4.5 $\mu \mathrm{M}$ (Durcan, Clarke, Magder \& Petri, 2015) and 0.5-0.7 $\mu \mathrm{M}$ (Miller, Fiechtner, Carpenter, Brown, Stroshane \& Stecher, 1987) for HCQ in SLE and rheumatoid arthritis patients on a daily $400 \mathrm{mg}$ dose. In contrast, its dose used in Covid-19, $1.2 \mathrm{~g} /$ day, would give substantially higher therapeutic concentrations of $\sim 10 \mu \mathrm{M}$ (Borba et al., 2020). Blood AZM levels following successive daily $500 \mathrm{mg}$ and two $250 \mathrm{mg}$ doses were reported at $0.22 \mu \mathrm{M}$ (Lai, Ho, Jain \& Walters, 2011). HCQ both alone and combined with AZM synergistically inhibited $\mathrm{I}_{\mathrm{Kr}}$ and $\mathrm{I}_{\mathrm{K} 1}$ with $\mathrm{IC}_{50} \mathrm{~s}$ at the high end of reported therapeutic levels, with some minor inhibition of $\mathrm{I}_{\mathrm{Na}}$ in this range.

Consistent with these experimental findings, computational molecular docking simulations utilizing a recent hERG homotetramer structure (PDB ID: 5va2) demonstrate potential HCQ binding. This is predominantly with Y652 and F656 from one or more hERG subunits. Additional residues involved in interactions with HCQ frequently included F557, L622, T623, S624, and S660. In contrast, the model predicted approximately two-fold weaker AZM-hERG binding.

Others have also explored the electrophysiological effects of HCQ or AZM on some cardiac ion channels but did so at three times the clinically relevant concentration and above (Capel et al., 2015; Lai, Ho, Jain \& Walters, 2011). Furthermore, the present study systematically evaluates HCQ and AZM in combination for the first time. Furthermore, the only report on electrophysiological effects of HCQ specifically studied sinoatrial cells, demonstrating significant reductions in hyperpolarization-activated currents $\left(\mathrm{I}_{\mathrm{f}}\right)$, with reduced $\mathrm{I}_{\mathrm{CaL}}$ and $\mathrm{I}_{\mathrm{Kr}}$ observed at $3 \mu \mathrm{M}$ (Capel et al., 2015). Nevertheless, this HCQ action on $\mathrm{I}_{\mathrm{f}}$ explains its bradycardic effects observed here, further illustrating its broad cardiac electrophysiological actions. Such a bradycardic medication in the presence of a hypotension accompanying sepsis or SARS could potentially exacerbate a septic shock.

At the whole heart level, our optical mapping studies demonstrate that HCQ correspondingly prolongs ventricular APD as well as $\mathrm{CaD}$, to extents further exacerbated by combination with AZM. This fulfils expectations from its inhibition of $\mathrm{I}_{\mathrm{Kr}}$ and $\mathrm{I}_{\mathrm{K} 1}$ and translates to the observed electrocardiographic QT prolongations. It is interesting to note that even prior to the Covid-19 pandemic, series of case reports have led to both HCQ and AZM being listed as definite causes of prolonged QT interval and risk of torsade de pointes for patients with long QT syndrome on crediblemeds.org (Roden, Harrington, Poppas \& Russo, 2020).

Prolonged APD predisposes to early afterdepolarisations (EADs) during the plateau phase of the ventricular action potential, classically attributed to $\mathrm{I}_{\mathrm{CaL}}$ reactivation (January \& Riddle, 1989). EADs are also more common under conditions of intracellular $\mathrm{Ca}^{2+}$ overload, including increased sympathetic drive: $\mathrm{I}_{\mathrm{CaL}}$ can be enhanced by high intracellular $\mathrm{Ca}^{2+}$ levels and by L-type $\mathrm{Ca}^{2+}$ channel phosphorylation by CaMKII(Herring, Kalla \& Paterson, 2019). EADs may potentially trigger ectopic beats and initiate arrhythmic events given suitable substrate.

In addition to these recovery phenomena, we also demonstrate for the first time that HCQ reduces ventricular conduction on multi-electrode arrays and optical mapping recording, and compromise atrioventricular and intraventricular conduction, reflected in prolonged PR intervals and QRS durations, to extents accentuated by further addition of AZM. Such findings are consistent with Na channel block (Reiz \& Nath, 1986) of the kind exemplified clinically by the CAST trials (Echt et al., 1991) and/or compromised gap junction function(King, Huang \& Fraser, 2013). Together, the combination demonstrated here of prolonged and 
increased dispersions of repolarisation and calcium handling combined with slowed conduction would offer a suitable substrate for sustained re-entrant arrhythmia. Thus, the combined exposure to HCQ and AZM, but not HCQ alone provoked alternans, ventricular tachycardic episodes, and re-entrant excitation following progressively accelerated cardiac pacing.

Finally, a validated human ventricular myocyte mathematical model integrated the observed ion channel properties with the effects on action potential and intracellular calcium handling characteristics in intact hearts. It separated whole heart electrophysiological features that were explicable in terms of the single channel properties and those attributable to altered calcium homeostasis. The modelling reproduced effects of surface ion channel function and their interactions forming a 'membrane clock' underlying the measurable electrical activity (Fig. 8C)(Lei \& Huang, 2019). It reproduced the action potential prolongation and consequent prolonged QT intervals with HCQ and the synergistic effects of combining HCQ with AZM, attributing these to their combined effects on $\mathrm{I}_{\mathrm{Kr}}$ and $\mathrm{I}_{\mathrm{K} 1}$. These latter events have been described as feeding forward into a ' $\mathrm{Ca}^{2+}$ clock' driving excitation-contraction coupling but whose events exert feedback effects modifying action potential conduction, recovery, and post-recovery stability, exemplified by $\mathrm{Ca}^{2+}$ feedback effects on $\mathrm{Na}^{+}$channel and gap junction function (Fig. 8C), as well as longer-term effects on channel expression(Huang, 2017; Lei, Wu, Terrar \& Huang, 2018). The modelling predicted the increased calcium transient magnitudes and durations particularly with HCQ+AZM (Fig 8C), but only small changes in maximum upstroke velocity attributable to $\mathrm{Na}$ channel blockade. The observed conduction slowing nevertheless could arise from a $\mathrm{Ca}^{2+}$-dependent gap junction uncoupling.

These results together provide an integrated basis for the electrophysiological and pharmacological effects of HCQ, AZM and their combination. They thereby underpin recent FDA guidelines cautioning against combined HCQ/AZM administration for treatment of Covid-19 on grounds of cardiac safety. Admittedly, physiological though not pro-arrhythmic effects occurred with HCQ administered alone at the $~ 10 \mu \mathrm{M}$ concentrations associated with Covid-19 therapy and not at the lower $\sim 1.0 \mu \mathrm{M}$ HCQ levels associated with therapy for rheumatoid arthritis and SLE. HCQ alone at therapeutic doses thus did not increase incidences of experimental ventricular arrhythmias in healthy hearts. Nevertheless, safety threshold doses could be reduced by accompanying cardiac injury arising from viral toxicity, sepsis, hypoxia-related myocyte injury, and immune-mediated cytokine storm in Covid-19. Furthermore, AZM potentiated the HCQ toxicity, now producing arrhythmic outcomes. The present findings thus strongly indicate a desirability for monitoring electrocardiographic, including QT, changes and cardiac injury during 4-aminoquinoline-macrolide therapy for Covid-19 patients.

\section{Sources of Funding}

This work is supported by the Kaifeng Excellent Key Laboratory Grant (Grant no. 20190601A) British Heart Foundation (BHF) (PG/14/79/31102, and PG/15/12/31280 and BHF Centres for Research Excellence (CRE) at Cambridge, CLHH, PG/14/80/31106, PG/16/67/32340, ML, PG. FS/17/52/33113,AT) and Oxford (ML) the Chinese Natural Science Foundation (31171085: ML) and EPSRC (United Kingdom) (EP/J00958X/1 and EP/I029826/1) (HZ).

\section{Disclosures}

The authors have declared that no conflict of interest exists.

\section{Author contributions}

GH, CL, AT, DP, CLHH, HZ and ML contributed to the study design

GW, XT, GH, YN, LW, XJ, GH contributed to the electrophysiological data acquisition.

GW, YD, GH, YX, LW, DL and NH contributed to the data analysis and visualization.

$\mathrm{HF}, \mathrm{KZ}, \mathrm{HZ}$ contributed to the action potential computer simulation. 
PM and ML contributed to the molecular docking computer simulation.

LW, DP, ML and GH contributed to the funding and resource acquisition.

ML and GH contributed to the supervision.

All authors contributed to the writing.

\section{References}

Borba MGS, Val FFA, Sampaio VS, Alexandre MAA, Melo GC, Brito M, et al. (2020). Effect of High vs Low Doses of Chloroquine Diphosphate as Adjunctive Therapy for Patients Hospitalized With Severe Acute Respiratory Syndrome Coronavirus 2 (SARS-CoV-2) Infection: A Randomized Clinical Trial. JAMA Network Open 3: e208857-e208857.

Capel RA, Herring N, Kalla M, Yavari A, Mirams GR, Douglas G, et al. (2015). Hydroxychloroquine reduces heart rate by modulating the hyperpolarization-activated current If: Novel electrophysiological insights and therapeutic potential. Heart Rhythm 12: 2186-2194.

Chorin E, Dai M, Shulman E, Wadhwani L, Bar-Cohen R, Barbhaiya C, et al. (2020). The QT interval in patients with COVID-19 treated with hydroxychloroquine and azithromycin. Nature Medicine. 10.1038/s41591-020-0888-2

Durcan L, Clarke WA, Magder LS, \& Petri M (2015). Hydroxychloroquine Blood Levels in Systemic Lupus Erythematosus: Clarifying Dosing Controversies and Improving Adherence. J Rheumatol 42:2092-2097.

Echt DS, Liebson PR, Mitchell LB, Peters RW, Obias-Manno D, Barker AH, et al. (1991). Mortality and morbidity in patients receiving encainide, flecainide, or placebo. The Cardiac Arrhythmia Suppression Trial. N Engl J Med 324: 781-788.

Gautret P, Lagier J-C, Parola P, Hoang VT, Meddeb L, Mailhe M, et al. (2020). Hydroxychloroquine and azithromycin as a treatment of COVID-19: results of an open-label non-randomized clinical trial. International Journal of Antimicrobial Agents: 105949.

Herring N, Kalla M, \& Paterson DJ (2019). The autonomic nervous system and cardiac arrhythmias: current concepts and emerging therapies. Nat Rev Cardiol 16: 707-726.

Hinton D (2020). Food and Drug Administration. FDA Emergency use authorization (EUA) of chloroquine and hydroxychloroquine. . Food and Drug Administration. .

Huang CL (2017). Murine Electrophysiological Models of Cardiac Arrhythmogenesis. Physiol Rev 97: 283409.

January CT, \& Riddle JM (1989). Early afterdepolarizations: mechanism of induction and block. A role for L-type Ca2+ current. Circ Res 64: 977-990.

King JH, Huang CL, \& Fraser JA (2013). Determinants of myocardial conduction velocity: implications for arrhythmogenesis. Front Physiol 4: 154.

Lai PC, Ho W, Jain N, \& Walters JD (2011). Azithromycin concentrations in blood and gingival crevicular fluid after systemic administration. J Periodontol 82: 1582-1586.

Lane JCE, Weaver J, Kostka K, Duarte-Salles T, Abrahao MTF, Alghoul H, et al. (2020). Safety of hydroxychloroquine, alone and in combination with azithromycin, in light of rapid wide-spread use for COVID-19: a multinational, network cohort and self-controlled case series study. medRxiv: 2020.2004.2008.20054551.

Lei M, \& Huang CL-H (2019). Cardiac arrhythmogenesis: a tale of two clocks? Cardiovascular Research. $10.1093 / \mathrm{cvr} / \mathrm{cvz} 283$

Lei M, Wu L, Terrar DA, \& Huang CL (2018). Modernized Classification of Cardiac Antiarrhythmic Drugs. Circulation 138: 1879-1896. 
Lenzer J (2020). Covid-19: US gives emergency approval to hydroxychloroquine despite lack of evidence. BMJ 369: m1335.

Magagnoli J, Narendran S, Pereira F, Cummings T, Hardin JW, Sutton SS, et al. (2020). Outcomes of hydroxychloroquine usage in United States veterans hospitalized with Covid-19. medRxiv:2020.2004.2016.20065920.

Mercuro NJ, Yen CF, Shim DJ, Maher TR, McCoy CM, Zimetbaum PJ, et al. (2020). Risk of QT Interval Prolongation Associated With Use of Hydroxychloroquine With or Without Concomitant Azithromycin Among Hospitalized Patients Testing Positive for Coronavirus Disease 2019 (COVID-19). JAMA Cardiology. 10.1001/jamacardio.2020.1834

Miller DR, Fiechtner JJ, Carpenter JR, Brown RR, Stroshane RM, \& Stecher VJ (1987). Plasma hydroxychloroquine concentrations and efficacy in rheumatoid arthritis. Arthritis Rheum 30: 567-571.

Negami T, Araki M, Okuno Y, \& Terada T (2019). Calculation of absolute binding free energies between the hERG channel and structurally diverse drugs. Sci Rep 9: 16586.

NIH (2020). NIH clinical trial of hydroxychloroquine, a potential therapy for COVID-19, begins.

O'Hara T, Virág L, Varró A, \& Rudy Y (2011). Simulation of the undiseased human cardiac ventricular action potential: model formulation and experimental validation. PLoS Comput Biol 7: e1002061.

Pharmafield (2020). Novartis sponsors hydroxychloroquine clinical trial in hospitalised COVID-19 patientsed. Morriss E.

Reiz S, \& Nath S (1986). Cardiotoxicity of local anaesthetic agents. Br J Anaesth 58: 736-746.

Roden DM, Harrington RA, Poppas A, \& Russo AM (2020). Considerations for Drug Interactions on QTc in Exploratory COVID-19 (Coronavirus Disease 2019) Treatment. Circulation. 10.1161/circulationaha.120.047521

Saleh M, Gabriels J, Chang D, Kim BS, Mansoor A, Mahmood E, et al. The Effect of Chloroquine, Hydroxychloroquine and Azithromycin on the Corrected QT Interval in Patients with SARS-CoV-2 Infection. Circulation: Arrhythmia and Electrophysiology 0. doi:10.1161/CIRCEP.120.008662

Strauss DG, Gintant G, Li Z, Wu W, Blinova K, Vicente J, et al.(2018). Comprehensive In Vitro Proarrhythmia Assay (CiPA) Update from a Cardiac Safety Research Consortium / Health and Environmental Sciences Institute / FDA Meeting. Therapeutic Innovation \& Regulatory Science 53: 519-525.

\section{Figures}

Figure 1. Effects of HCQ on ion currents attributable to hNav1.5 ( $\left.\mathrm{I}_{\mathrm{Na}}, \mathrm{A}\right)$, Cav1.2 ( $\left.\mathrm{I}_{\mathrm{Ca}, \mathrm{L}}, \mathrm{B}\right), \mathrm{Kv} 4.3$ ( $\mathrm{I}_{\mathrm{to}}, \mathrm{C}$ ), hERG ( $\left.\mathrm{I}_{\mathrm{Kr}}, \mathrm{D}\right), \mathrm{KCNQ} 1 / \mathrm{E} 1\left(\mathrm{I}_{\mathrm{Ks}}, \mathrm{E}\right)$ and Kir2.1 ( $\left.\mathrm{I}_{\mathrm{K} 1}, \mathrm{~F}\right)$. (A-F) Left panels (i): Typical currents in the presence of $0,1,10$ and $100 \mu \mathrm{M}$ HCQ. Insets summarize the voltage clamping protocols. Right panels (ii): Mean concentration-response plots constructed for the maximum magnitude of each current (n cells).

Figure 2. Effects of AZM on ion currents attributable to hNav1.5 ( $\left.\mathrm{I}_{\mathrm{Na}}, \mathrm{A}\right)$, Cav1.2 ( $\left.\mathrm{I}_{\mathrm{Ca}, \mathrm{L}}, \mathrm{B}\right), \mathrm{Kv} 4.3$ ( $\mathrm{I}_{\mathrm{to}}, \mathrm{C}$ ), hERG $\left(\mathrm{I}_{\mathrm{Kr}}, D\right)$, KCNQ1/E1 ( $\left.\mathrm{I}_{\mathrm{Ks}}, \mathrm{E}\right)$ and Kir2.1 ( $\left.\mathrm{I}_{\mathrm{K} 1}, \mathrm{~F}\right)$. (A-F) Left panels (i): Typical currents in the presence of $0,1,10$ and $100 \mu \mathrm{M}$ HCQ. Insets summarize the voltage clamping protocols. Right panels (ii): Mean concentration-response plots constructed for the maximum magnitude of each current ((n cells).

Figure 3. Effects of HCQ in combination with $10 \mu \mathrm{M}$ AZM on ionic currents attributable to hNav1.5 ( $\mathrm{I}_{\mathrm{Na}}$, A), Cav1.2 ( $\left.\mathrm{I}_{\mathrm{Ca}, \mathrm{L}}, \mathrm{B}\right), \operatorname{Kv} 4.3\left(\mathrm{I}_{\mathrm{to}}, \mathrm{C}\right)$, hERG $\left(\mathrm{I}_{\mathrm{Kr}}, \mathrm{D}\right)$, KCNQ1/E1 ( $\left.\mathrm{I}_{\mathrm{Ks}}, \mathrm{E}\right)$ and Kir2.1 ( $\left.\mathrm{I}_{\mathrm{K} 1}, \mathrm{~F}\right)$. (A-F) Left panels (i): Typical currents in the presence of $0,1,10$ and $100 \mu \mathrm{M}$ HCQ with $10 \mu \mathrm{M}$ AZM. Insets summarize the voltage clamping protocols. Right panels (ii): Mean concentration-response plots constructed for the maximum magnitude of each current (n cells). 
Figure 4. Computational docking studies. (A) hERG homotetramer structure (PDB ID: 5va2) with HCQ molecule (space-filling representation) indicating binding site placement. Residues 544-671 used in IFD of hERG chains A, B, C, and D colored red, green, yellow, and blue, respectively. (B) Overlay of IFD HCQ positions. (C) Representative, top-scoring positions of HCQ (ball-and-stick). Y652 and F656 residues in stick representation colored accordingly to their parent chains. (D) 2-dimensional (2D) diagram depicting interaction types between presented HCQ position and hERG. (E) Representative, top-scoring position of AZM, and (F) 2D diagram depicting AZM-hERG interactions. Diagrams were prepared using Non-bond Interactions Monitor tool in Discovery Studio Visualizer (Dassault Systèmes, Vélizy-Villacoublay, France). IFD = Induced Fit Docking.

Figure 5. Multi-electrode array (MEA) mapping and electrocardiographic (ECG) studies in isolated perfused guinea-pig hearts following HCQ and AZM challenge. (A) Schematic summary of stimulation and MEA and ECG recording configuration. (B) Typical left atrial and left ventricular isochronal conduction maps obtained in the presence of $0,0.01,0.1,1.0$ and $10 \mu \mathrm{M} \mathrm{HCQ}$. (C) (i) Successive LV isochronal maps before and following addition of $10 \mu \mathrm{M} \mathrm{HCQ}$ and $10 \mu \mathrm{M} \mathrm{HCQ}$ and $10 \mu \mathrm{M}$ AZM in combination followed by drug washout. (ii) Quantified conduction velocities through these conditions. (D) ECG studies: (i) ECG records obtained before and following application of $10 \mu \mathrm{M} \mathrm{HCQ}$, and $10 \mu \mathrm{M}$ HCQ and $10 \mu \mathrm{M}$ AZM combined, followed by drug washout. (ii)-(v): corresponding measured (ii) heart rates (HR), (iii) QT and (iv) PR intervals and (v) QRS durations (n hearts) ${ }^{*} \mathrm{p}<0.05,{ }^{* *} \mathrm{p}<0.01,{ }^{* * *} \mathrm{p}<0.001$

Figure 6. Voltage RH237 and Rhod-2 optical mapping in isolated perfused hearts. Comparisons of results before and following challenge by $1 \mu \mathrm{M}$ and $10 \mu \mathrm{M} \mathrm{HCQ}$, and $10 \mu \mathrm{M}$ HCQ combined with either 1 or $10 \mu \mathrm{M}$ AZM. (A, B) RH237 mapping: (A)(i) Optical mapping and ECG monitoring configuration (ii) Maps of action potential initiation and conduction; (iii) heart rates and (iv) conduction velocities. (B) Action potential timecourses and recoveries: (i) Maps of AP durations at 90\% repolarization ( $\mathrm{APD}_{90}$ ). (ii) Representative APs recorded from defined regions of interest. (iii) $\mathrm{APD}_{90} \mathrm{~s}$ averaged over the field of view. (C) Rhod-2 AM $(\mathrm{CaD})$ mapping of ventricular $\mathrm{Ca}^{2+}$ transients $(\mathrm{CaT})$ : (i) maps of $\mathrm{CaD}$ durations at $90 \%$ recovery $\left(\mathrm{CaD}_{90}\right)$. (ii) Comparison of typical CaT transients averaged over defined regions of interest; (iii) $\mathrm{CaD}_{90}$ magnitudes; (iv) $\mathrm{CaD}_{90}$ dispersions ( $\mathrm{n}$ hearts). NS not significant, ${ }^{*} \mathrm{p}<0.05,{ }^{* *} \mathrm{p}<0.01,{ }^{* * *} \mathrm{p}<0.001$.

Figure 7. Voltage RH237 optical mapping of re-entry and ventricular arrhythmic tendency in isolated perfused hearts. (A) Recording and programmed stimulation configuration. (B) Optical action potential traces obtained under programmed pacing at $190 \mathrm{~ms}, 180 \mathrm{~ms}$ and $170 \mathrm{~ms}$ cycle lengths (CLs), before (control) and following addition of $10 \mu \mathrm{M} \mathrm{HCM}$, and $10 \mu \mathrm{M}$ HCM with added $10 \mu \mathrm{M}$ AZM. (C) Corresponding voltage maps obtained at the numbered colour-coded timepoints (n hearts).

Figure 8. In silico modelling of action potentials before and following HCQ and combined HCQ and AZM challenge. (A) Simulated action potentials of human ventricular cell under control conditions and in the presence of HCQ $(10 \mu \mathrm{M})$ and HCQ $(10 \mu \mathrm{M})+$ AZM $(1 \mu \mathrm{M})$ combined. HCQ and AZM when combined produced a synergistic action in prolonging action potential duration $\left(\mathrm{APD}_{90}\right)$, as well as reducing the repolarization rate following the end of phase 0 depolarisation. (B) Effects of HCQ and HCQ + AZM combined on $\mathrm{APD}_{90}$ and the maximal upstroke velocity (MUV) of the AP. (C) Illustration of electrophysiological effects and possible mechanisms underlying the pro-arrhythmic effects of applications of HCQ and HCQ and AZM combined at the ion channel, cellular and tissue levels. 
A $\mathrm{Na}_{\mathrm{v}} 1.5$
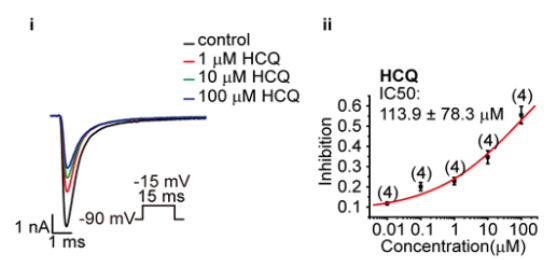

B $\mathrm{Ca}_{\mathrm{v}} 1.2$

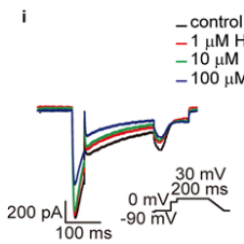

C $\mathrm{K}_{\mathrm{v}} 4.3$
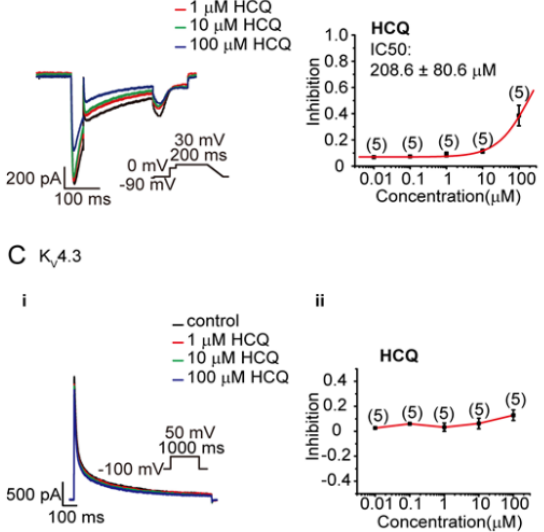

D hERG

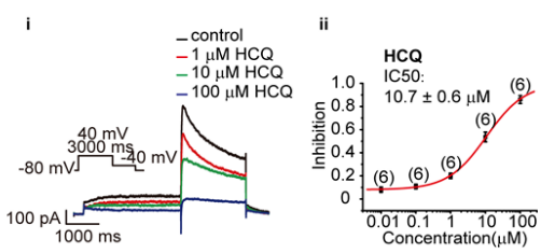

E KCNQ1/E1

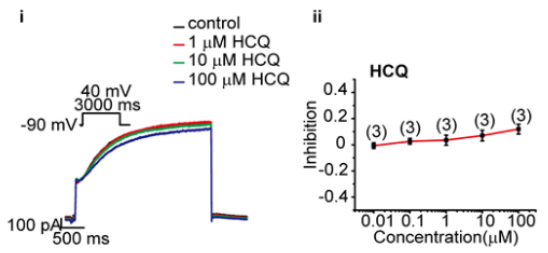

F Kir2.1
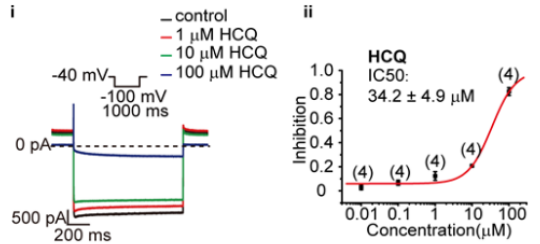

A Na 1.5
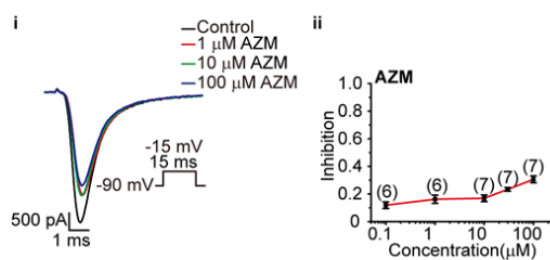

B $\quad \mathrm{Ca}_{\mathrm{v}} 1.2$
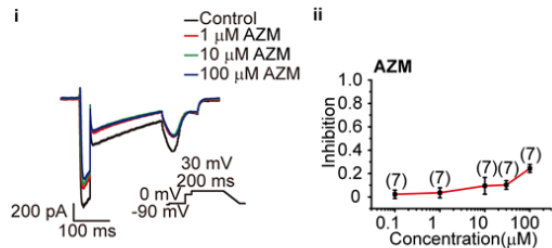

C $\mathrm{k}_{\mathrm{v}} 4.3$

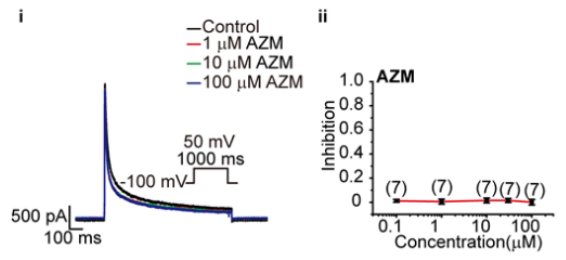

D hERG

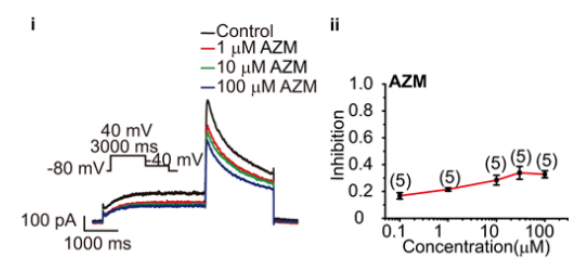

E KCNQ1/E1

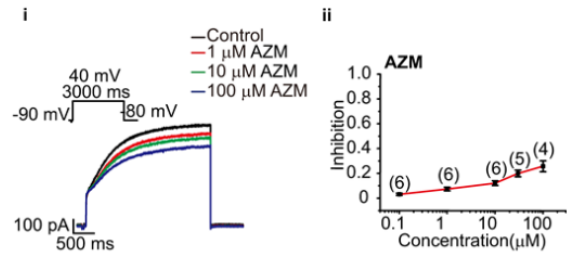

$F_{\text {Kir2.1 }}$

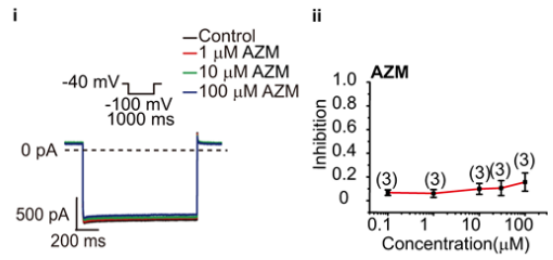


A $\mathrm{Na}_{v} 1.5$

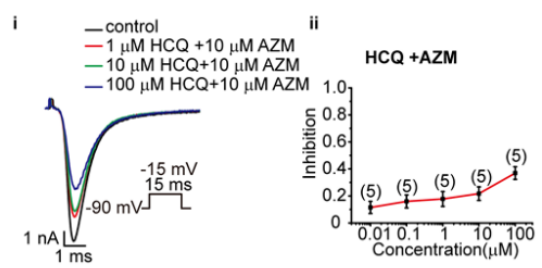

B $\quad \mathrm{Ca}_{\mathrm{v}} 1.2$

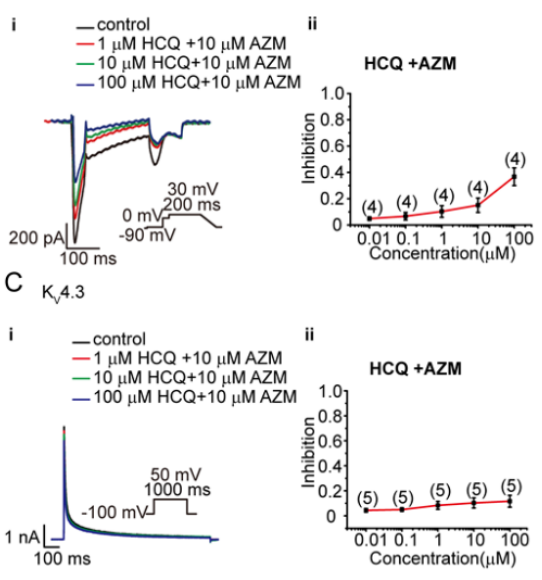

$D_{\text {hERG }}$

i $\quad$ control $1 \mu \mathrm{M} \mathrm{HCQ}+10 \mu \mathrm{M} \mathrm{AZM}$ ii $-10 \mu \mathrm{M} \mathrm{HCQ}+10 \mu \mathrm{MAZM}$
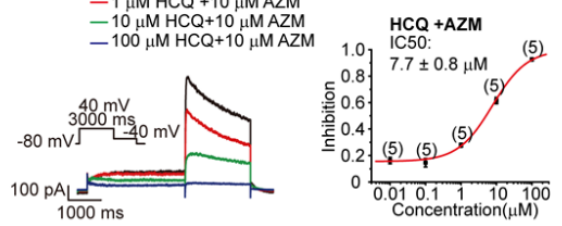

E KCNQ1/E1

i $\quad$ control $1 \mu \mathrm{M} \mathrm{HCQ}+10 \mu \mathrm{M} \mathrm{AZM}$ ii $-10 \mu \mathrm{M} \mathrm{HCQ}+10 \mu \mathrm{MAZM}$ $-10 \mu \mathrm{M} H C Q+10 \mu M \mathrm{AZM} \quad \mathrm{HCQ}+\mathrm{AZM}$

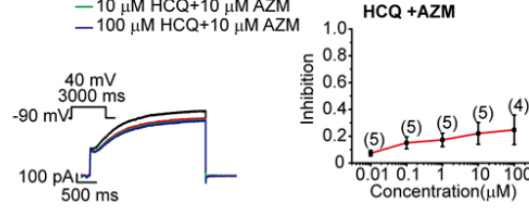

$\mathrm{F}_{\text {Kir2.1 }}$

i $\quad$ control $1 \mu \mathrm{M} \mathrm{HCQ}+10 \mu \mathrm{M} \mathrm{AZM} \mathrm{ii}$ $-10 \mu \mathrm{M} \mathrm{HCQ}+10 \mu \mathrm{M} \mathrm{AZM}$

HCQ + AZM $-100 \mu \mathrm{M} \mathrm{HCQ}+10 \mu \mathrm{M}$ AZM 1.0 IC50:

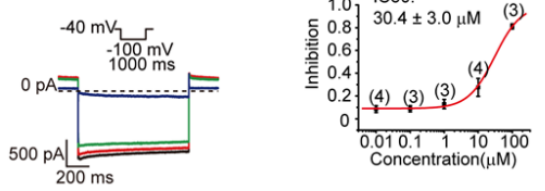



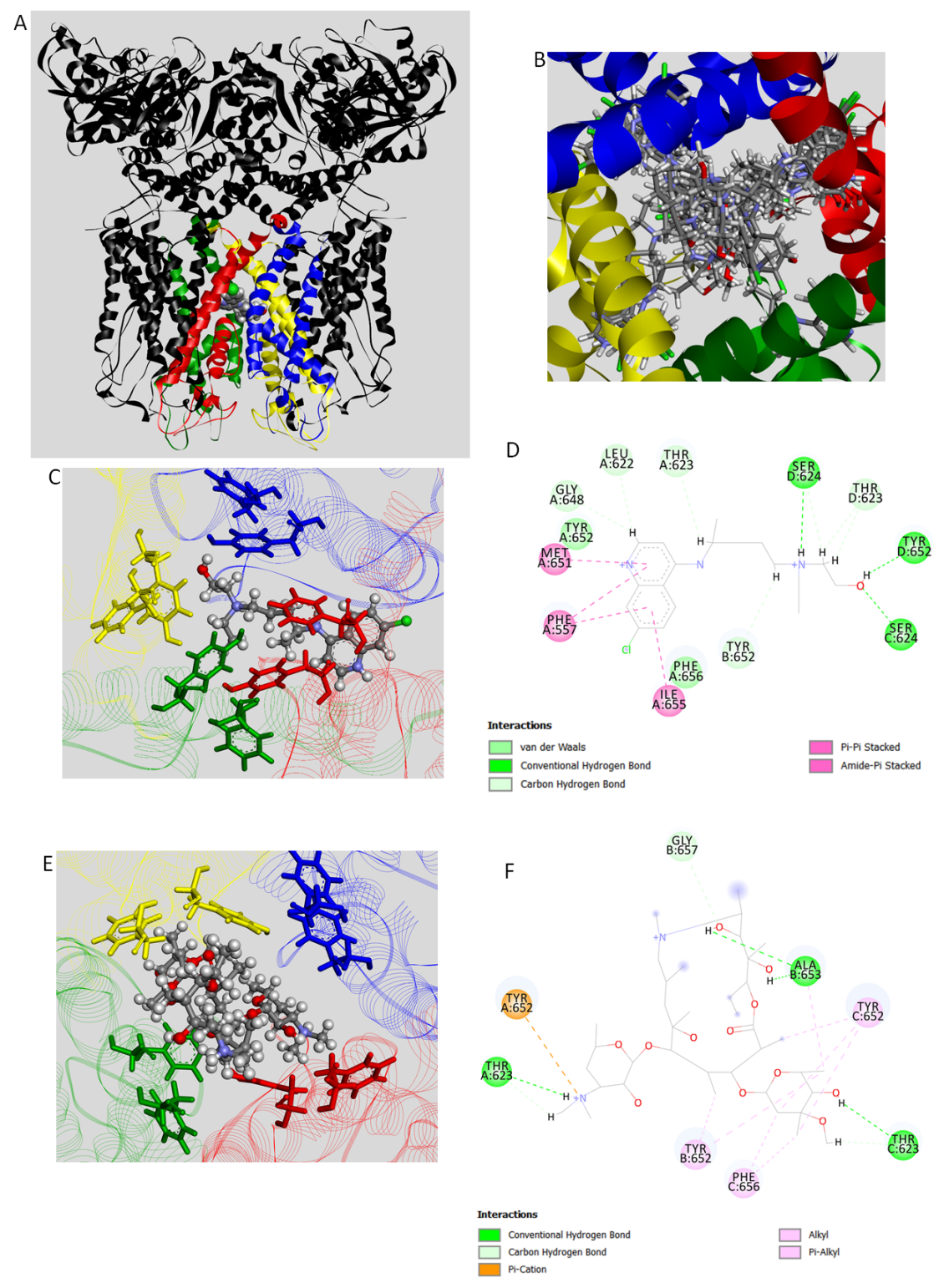
A

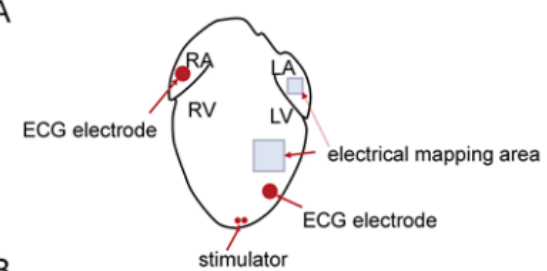

B

Isochronal maps

LA

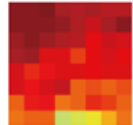

LV

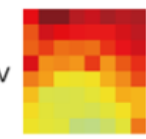

C
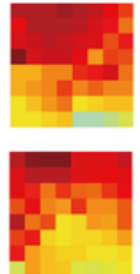

HCQ $0.01 \mu \mathrm{M} \quad \mathrm{HCQ} 0.1 \mu \mathrm{M}$
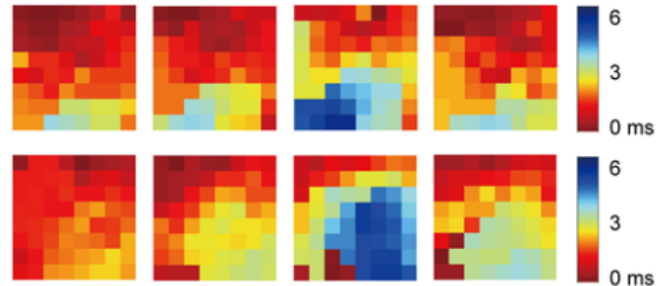

$\mathrm{HCQ} 1 \mu \mathrm{M}$

HCQ $10 \mu \mathrm{M}$

Wash
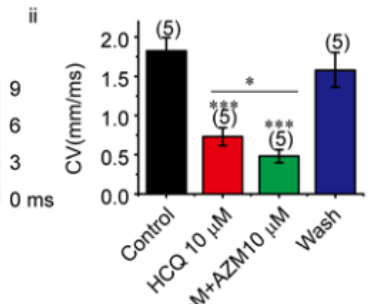

D
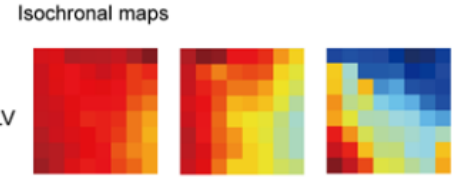

HCQ $10 \mu \mathrm{M}$

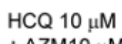
$+\mathrm{AZM} 10 \mu \mathrm{M}$

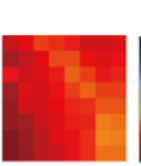

Wash

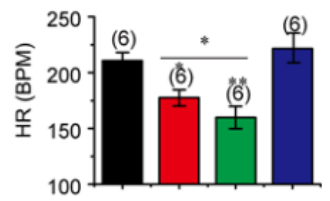

iii

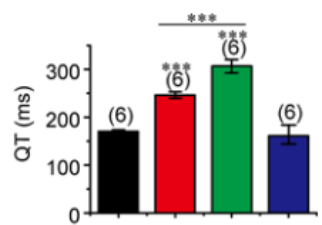

$\mathrm{HCQ} 10 \mu \mathrm{M}+\mathrm{AZM} 10 \mu \mathrm{M}$

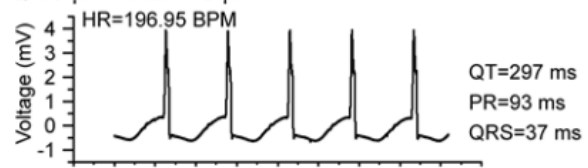

iv

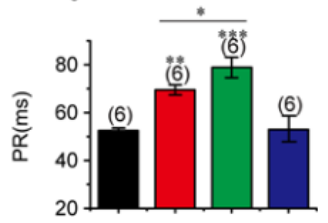

Wash
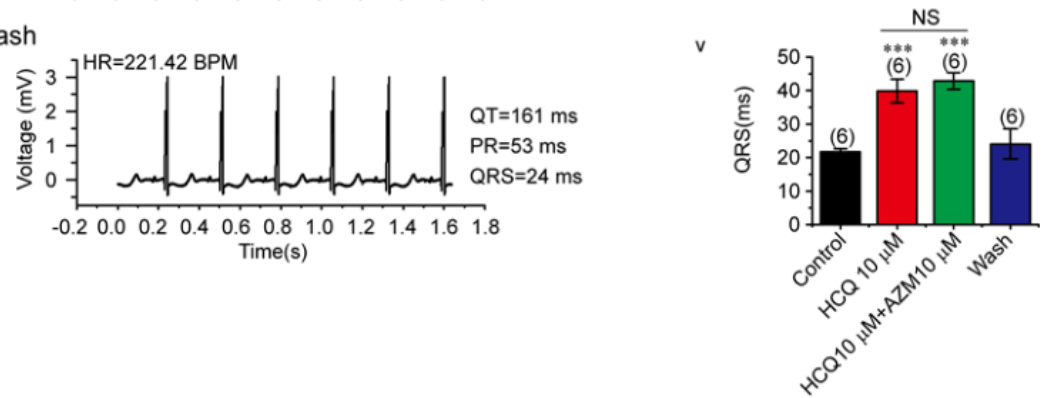
A

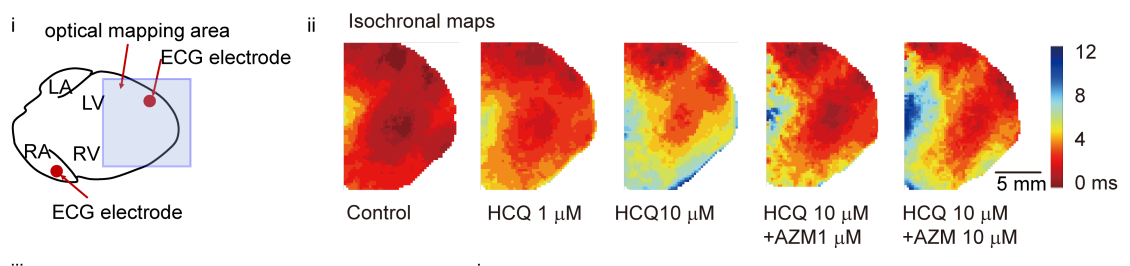

iii
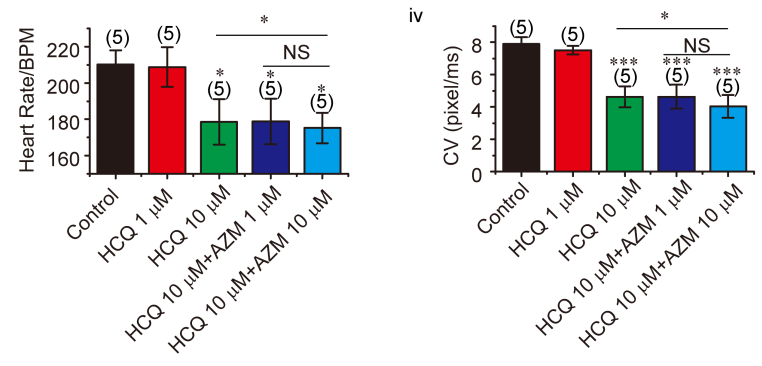

B
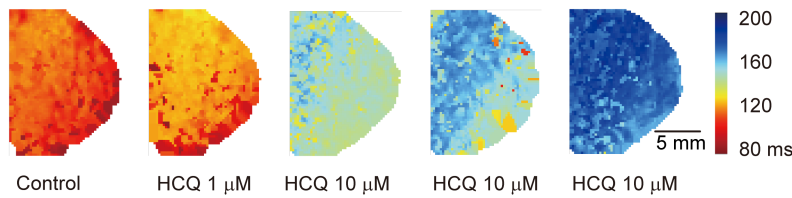

$+A Z M 1 \mu M$

+AZM $10 \mu \mathrm{M}$

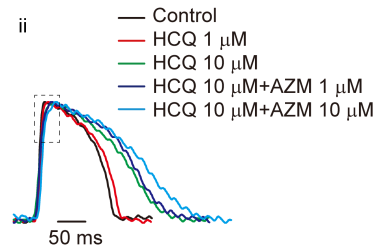

iii

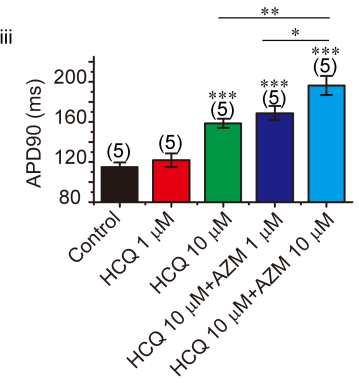

C
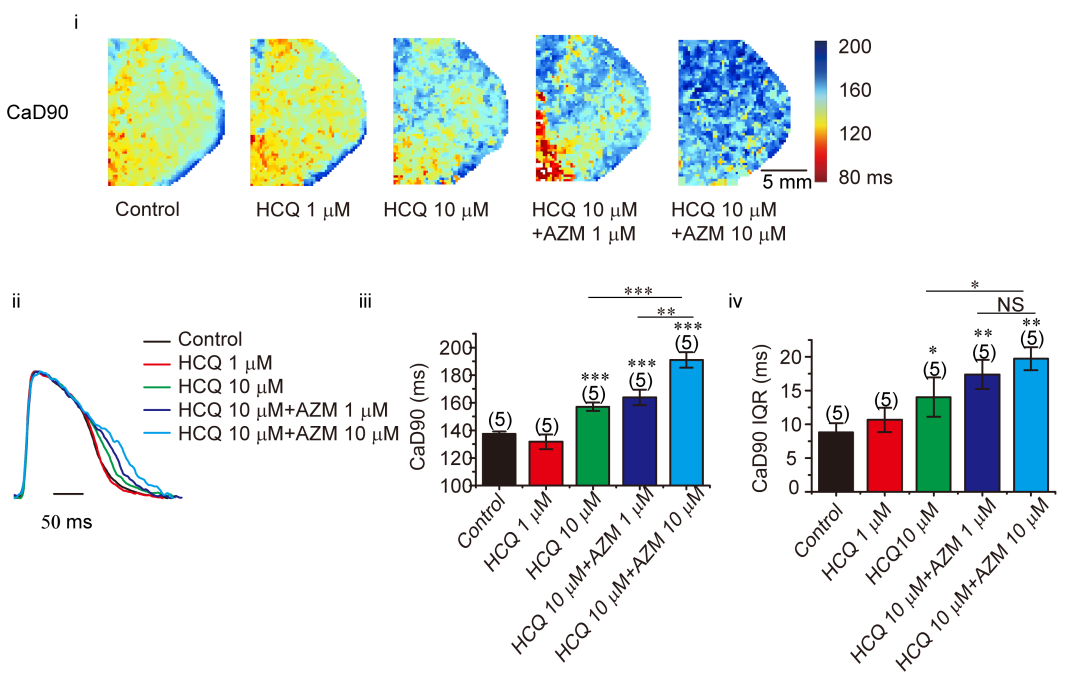
A

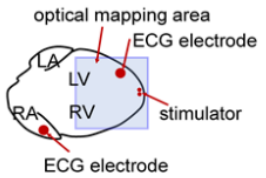

B

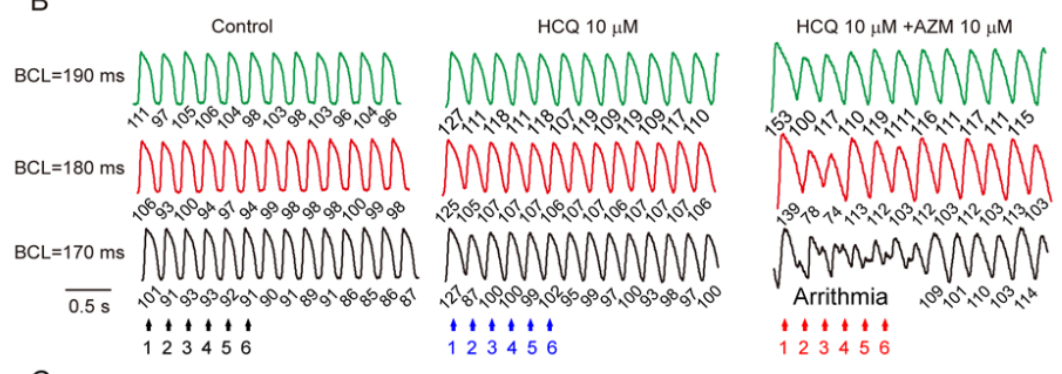

C
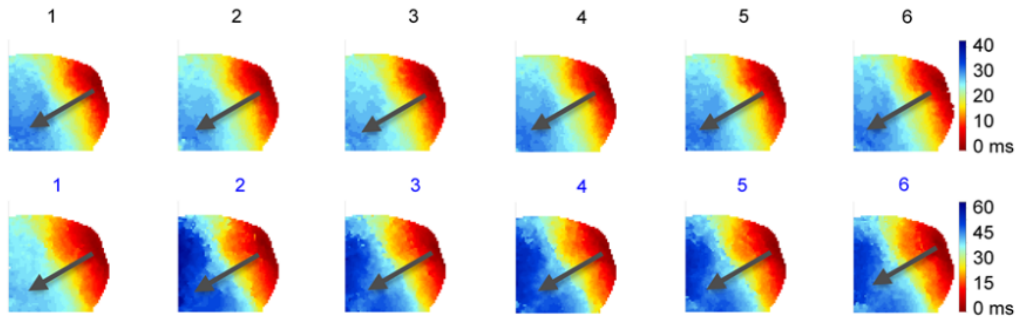

6
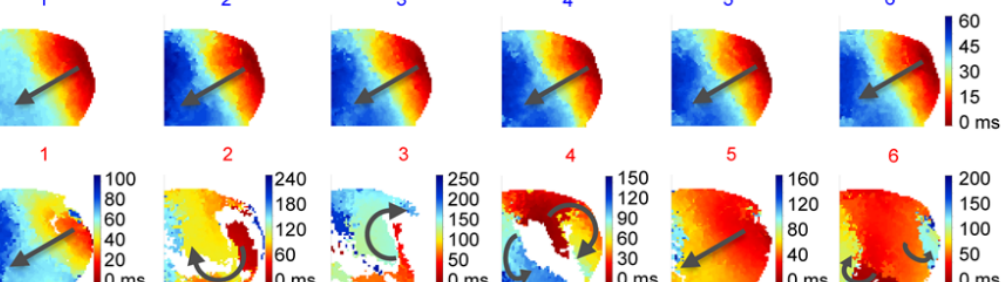

4

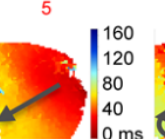

6

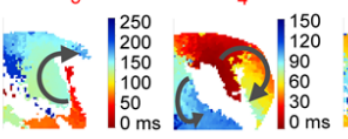




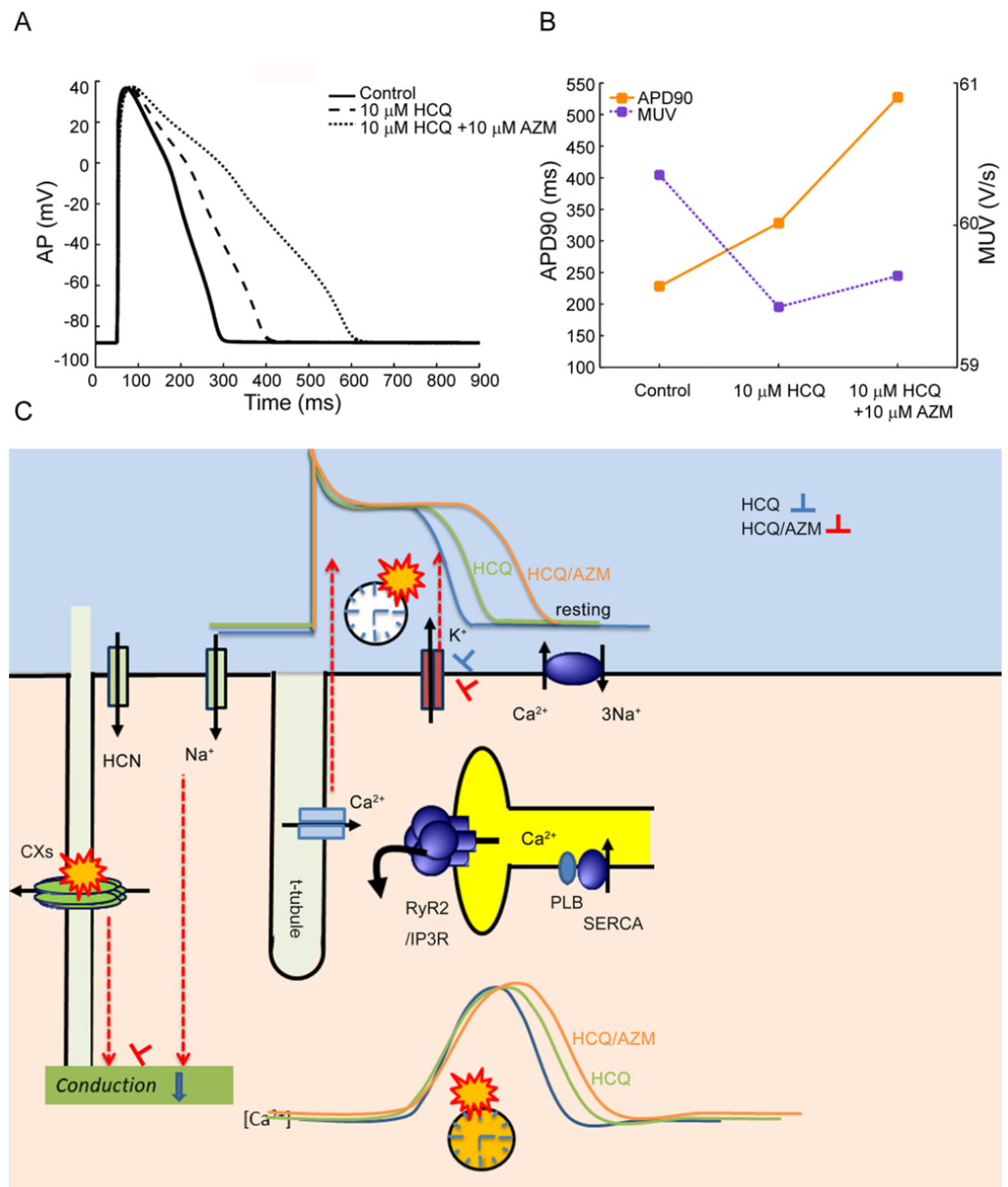

